\title{
COMPUTATION OF STRESS INTENSITY FACTORS BY THE SUB-REGION MIXED FINITE ELEMENT METHOD OF LINES *
}

\author{
Yuan Si \\ ( Department of Civil Engineering, Tsinghua University, Beijing 100084, China) \\ $\mathrm{Xu}$ Yongjun \\ ( Institute of Mechanics, Chinese Academy of Sciences, Beijing 100080, China) \\ WILLIAMS F W \\ ( Structural Engineering Division, Cardiff University, Cardiff CF2 3TB, UK)
}

Received 26 July 2006; revision received 12 April 2007

\begin{abstract}
Based on the sub-region generalized variational principle, a sub-region mixed version of the newly-developed semi-analytical 'finite element method of lines' (FEMOL) is proposed in this paper for accurate and efficient computation of stress intensity factors (SIFs) of two-dimensional notches/cracks. The circular regions surrounding notch/crack tips are taken as the complementary energy region in which a number of leading terms of singular solutions for stresses are used, with the sought SIFs being among the unknown coefficients. The rest of the arbitrary domain is taken as the potential energy region in which FEMOL is applied to obtain approximate displacements. A mixed system of ordinary differential equations (ODEs) and algebraic equations is derived via the sub-region generalized variational principle. A singularity removal technique that eliminates the stress parameters from the mixed equation system eventually yields a standard FEMOL ODE system, the solution of which is no longer singular and is simply and efficiently obtained using a standard general-purpose ODE solver. A number of numerical examples, including bi-material notches/cracks in anti-plane and plane elasticity, are given to show the generally excellent performance of the proposed method.
\end{abstract}

KEY WORDS stress intensity factors, finite element method of lines, sub-region generalized variational principle, ordinary differential equation solver

\section{INTRODUCTION}

Stress analysis in engineering applications inevitably encounters stress singularities caused by sudden changes in geometry, e.g. around re-entrant corners (notches) or, more severely, around crack tips. Their presence causes great difficulty in obtaining the numerical solutions, which have to be invoked when analytical solutions are not available. A numerical recipe for accurate and efficient computation of stress singularity factors (SIFs) usually consists of two major ingredients, namely, a powerful numerical method for general stress analysis and a novel approach to obtaining the desired SIFs which may involve special

\footnotetext{
* Project supported by the National Natural Sciences Foundation of China (Nos. 59525813 and 19872066) and the Cardiff Advanced Chinese Engineering Centre of Cardiff University.
} 
treatment of various singularities. Instead of giving an extensive review of various existing numerical approaches, our discussion is confined to a brief introduction to the major ingredients adopted in the present paper.

The underlying numerical method employed in this paper is the finite element method of lines $(\text { FEMOL })^{[1-4]}$, which is a newly-developed, general-purpose, semi-analytical one. In this method, a partial differential equation defined in an arbitrary domain is semi-discretized, by finite element techniques via energy theorems or variational principles, into a system of ordinary differential equations (ODEs) defined on straight or curved mesh lines. At present, the resulting ODE system is directly and efficiently solved by a state-of-the-art ODE solver, e.g. COLSYS ${ }^{[5,6]}$ is exclusively adopted in the present paper. Such solvers have built-in self-adaptivity features such that the accuracy of the ODE solutions satisfies the user pre-specified error tolerances. With these solvers, FEMOL has proved itself a remarkable numerical method with efficient automatically built-in adaptivity in the mesh line directions, so that its inherent semi-analytical characteristics are well preserved. Its power and versatility have been demonstrated by a series of theoretical analyses and computational applications to various linear and nonlinear problems. A general-purpose computer code FEMOL92 ${ }^{[7]}$ capable of static and vibratory analysis of various linear elastic structures has been developed. For a more detailed and systematic description of FEMOL see Ref.[4].

FEMOL could be directly applied to notch/crack problems ${ }^{[3,4]}$. The success in establishing "triangular' elements ${ }^{[8]}$ by degenerating an element end-side to a point allows the use of the optimum radial line meshes, i.e., mesh lines emanate from crack tips. With no special treatment, except for judicious use of radial line meshes with sufficiently high polynomial degree $p$ used in the element displacement shape functions, FEMOL is able to produce remarkably accurate solutions even when very few elements are used ${ }^{[4]}$. However, such successful computation has a price because the ODE solver employed, which is responsible for the singularity solutions on these radially arranged mesh lines, needs heavy computational effort with respect to adaptivity to capture the local singularity behavior. For instance, the code COLSYS, which uses a spline collocation algorithm at Gaussian points, would progressively impose far more collocation points near the singular end-point than it does for regular problems. Moreover, even a moderately stringent tolerance for the ODE solutions may cause the computer resources to be exhausted.

While direct FEMOL analysis of singular problems avoids using any analytical singular solutions near crack tips, additional computational effort is required to extract the desired SIFs from the computed numerical solutions and hence, for most existing extraction methods, knowledge of the analytical singular solutions has to be used, usually in an eigen-function expansion form. Xu and Yuan et al. ${ }^{[0-14]}$ presented some effective methods for accurate and reliable computation of complete real or complex eigen-solutions in two-dimensional notch/crack singularities with multiple materials, arbitrary opening angles and various surface conditions. The resulting algorithm is robust and may be employed by any numerical methods that make use of singular solutions.

In order to take full advantage of the power of FEMOL and the usefulness of the local singular solutions, the sub-region generalized variational principle is utilized in this paper to lay a theoretical foundation for the proposed implementation and to facilitate the integration of the two solution components. This variational principle was first presented by Long ${ }^{[15]}$ and was extended by Long et al. ${ }^{[16]}$. It soon found successful applications in the finite element (FE) analysis of singularity problems, especially in SIF computations $^{[17-19]}$. A major problem in standard FE implementation is the uncertainty in choosing the size of the singular region and hence the sensitivity of the computed results to the size of the singular region. This is mainly due to a lack of the adaptivity feature in standard FE implementation.

The present paper presents a natural extension of the application of the sub-region generalized variational principle to the newly-developed FEMOL for accurate and efficient SIF computations. In the proposed method, a circular singular region which contains the notch/crack tip as a sector, see $\Omega_{C}$ in Fig.1(b), is taken as the complementary energy region in which a number of leading terms of singular solutions for stresses are used, with the sought SIFs being part of the unknown coefficients. The rest of the arbitrary domain, i.e., $\Omega_{P}$ in Fig.1(b), is taken as the potential energy region in which FEMOL is applied to obtain highly accurate displacements. By applying the sub-region generalized variational principle, a mixed system of ODEs and algebraic equations is derived, which cannot be solved by a standard ODE solver directly. A singularity removing technique is proposed to eliminate the singularity 
coefficients from the mixed equation system and hence yields a standard FEMOL ODE system, which can be directly and efficiently solved by COLSYS. A subsequent back substitution immediately gives the solution for the singularity coefficients, which can be converted into SIFs by simple calculations. Because of the semi-analytical nature of FEMOL, the computed results are more accurate and less sensitive to the size of the singular region than the FEM solutions.

The anti-plane strain and plane stress/strain problems are considered in this paper. In both cases, without losing generality, body forces are not considered and only homogeneous displacement boundary conditions are taken into account. For the plane elasticity problem, only the plane stress case is discussed below and the two cases are simply called the anti-plane and plane stress cases in this paper. A number of illustrative numerical examples, including bi-material notches/cracks, are given to show the generally excellent performance of the proposed method.

\section{SUB-REGION MIXED ENERGY FUNCTIONAL}

Consider an arbitrary two-dimensional domain $\Omega$ containing a notch in $N$ different materials, as shown in Fig.1(a). It is partitioned into two sub-regions $\Omega_{C}$ and $\Omega_{P}$, of which the former is a singular region and is judiciously chosen to be circular with the notch/crack tip at its centre, and the latter is the remaining regular region, see Fig.1(b). The stresses in $\Omega_{C}$ and the displacements in $\Omega_{P}$ are chosen as the basic unknowns. Accordingly, the total complementary energy $\Pi_{C}$ in $\Omega_{C}$ (excluding the interface $\Gamma_{P C}$ ), the total potential energy $\Pi_{P}$ in $\Omega_{P}$ (also excluding the interface $\Gamma_{P C}$ ) and the work $H_{P C}$ done on the interface $\Gamma_{P C}$ by the stresses in $\Omega_{C}$ on the displacements in $\Omega_{P}$ will be dealt with. When emphasis is on the different energy functionals, the regions $\Omega_{C}$ and $\Omega_{P}$ will be called the $C$ - and $P$-regions, respectively.

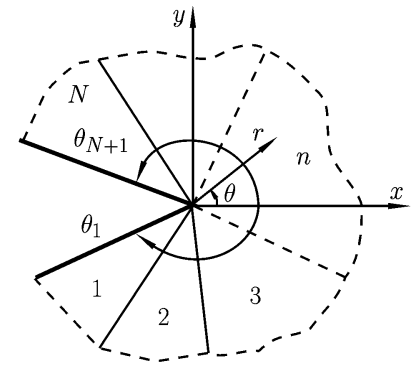

(a) original problem

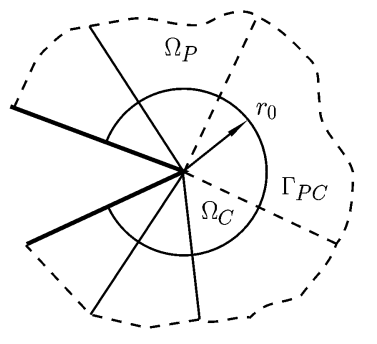

(b) sub-region FEMOL mesh

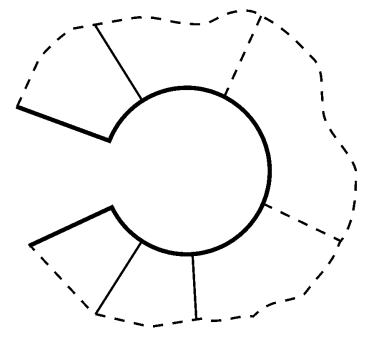

(c) FEMOL region with singularity removal technique

Fig. 1. $N$-material notch problem

The sub-region FEMOL formulation is based on the sub-region generalized variational principle ${ }^{[15,16]}$, which states that for the true solution the following sub-region mixed energy functional has a stationary value

$$
\Pi=\Pi_{P}-\Pi_{C}+H_{P C}
$$

For the anti-plane case, the three terms in Eq.(1) can be respectively expressed as

$$
\begin{aligned}
& \Pi_{P}=\sum \frac{G_{n}}{2} \iint_{\Omega_{P n}}\left[\left(\frac{\partial w}{\partial x}\right)^{2}+\left(\frac{\partial w}{\partial y}\right)^{2}\right] \mathrm{d} x \mathrm{~d} y-\int_{\Gamma_{S}} w \bar{q}_{z} \mathrm{~d} s \\
& \Pi_{C}=\sum \frac{1}{2 G_{n}} \iint_{\Omega_{C n}}\left(\tau_{r z}^{2}+\tau_{\theta z}^{2}\right) r \mathrm{~d} r \mathrm{~d} \theta \\
& H_{P C}=\sum \int_{\Gamma_{P C n}} \tau_{r z} w \mathrm{~d} s
\end{aligned}
$$

where $x y z$ is a right-hand Cartesian axis system, the summation is over each material $n, G_{n}$ is the shear modulus of material $n, w$ is the displacement in the $P$-region, $\Gamma_{S}$ is the boundary section on which traction force $\bar{q}_{z}$ is prescribed and, $\tau_{r z}$ and $\tau_{\theta z}$ are stress components in the $C$-region. 
For the plane stress case, the three terms in Eq.(1) can be expressed in matrix form as

$$
\begin{aligned}
& \Pi_{P}=\sum \frac{1}{2} \iint_{\Omega_{P n}}\{\varepsilon\}^{\mathrm{T}}[D]\{\varepsilon\} \mathrm{d} x \mathrm{~d} y-\int_{\Gamma_{S}}\{\bar{q}\}^{\mathrm{T}}\{u\} \mathrm{d} s \\
& \Pi_{C}=\sum \frac{1}{2} \iint_{\Omega_{C n}}\{\sigma\}^{\mathrm{T}}[D]^{-1}\{\sigma\} r \mathrm{~d} r \mathrm{~d} \theta \\
& H_{P C}=\sum \int_{\Gamma_{P C_{n}}}\left(\sigma_{r} u_{r}+\tau_{r \theta} u_{\theta}\right) \mathrm{d} s
\end{aligned}
$$

where

$$
\{\sigma\}=\left\{\begin{array}{c}
\sigma_{r} \\
\sigma_{\theta} \\
\tau_{r \theta}
\end{array}\right\}, \quad\{\varepsilon\}=\left\{\begin{array}{l}
\varepsilon_{x} \\
\varepsilon_{y} \\
\gamma_{x y}
\end{array}\right\}, \quad[D]=\frac{E}{1-\nu^{2}}\left[\begin{array}{ccc}
1 & \nu & 0 \\
\nu & 1 & 0 \\
0 & 0 & \frac{1-\nu}{2}
\end{array}\right], \quad\{u\}=\left\{\begin{array}{l}
u_{x} \\
u_{y}
\end{array}\right\}, \quad\{\bar{q}\}=\left\{\begin{array}{l}
\bar{q}_{x} \\
\bar{q}_{y}
\end{array}\right\}
$$

and the symbols have their usual meanings.

\section{THE COMPLEMENTARY ENERGY REGION}

In the complementary energy region, the stresses are assumed to be of the form of the Williams eigen-function expansion. The required eigen-values i.e., the $\lambda_{i}$ 's, may be complex in the plane stress case and are found by using the embedding algorithm ${ }^{[9]}$, which reliably gives numerically exact results. 3.1. The Anti-plane Case

In the polar coordinates shown in Fig.1(a), the singular solution of the Williams type expansion in the $C$-region takes the following general form in each material domain

$$
w_{n}=\sum_{i=1}^{\infty} \alpha_{i} r^{\lambda_{i}} f_{i}^{n}(\theta), \quad f_{i}^{n}(\theta)=a_{i}^{n} \cos \left(\lambda_{i} \theta\right)+b_{i}^{n} \sin \left(\lambda_{i} \theta\right), \quad 1 \leq n \leq N
$$

From the stress-free condition for the notch/crack surfaces and the compatibility conditions for interfaces between different materials, all of the coefficients $a_{i}^{n}$ and $b_{i}^{n}$ for the different materials $(1 \leq n \leq N)$ can be expressed in terms of a single coefficient, namely, $\alpha_{i}$. Therefore a $k$ term singular solution can be written in matrix form as follows:

$$
w_{n}=\sum_{i=1}^{k} \alpha_{i} r^{\lambda_{i}} f_{i}^{n}(\theta)=\left\{S^{n}\right\}^{\mathrm{T}}\{\alpha\}, \quad\{\alpha\}^{\mathrm{T}}=\left\{\begin{array}{llll}
\alpha_{1} & \alpha_{2} & \ldots & \alpha_{k}
\end{array}\right\}
$$

which after differentiation provides a natural and ideal representation of the admissible stresses in the $C$-region as

$$
\tau_{r z}^{n}=G_{n} \frac{\partial w_{n}}{\partial r}=G_{n}\left\{S_{r}^{n}\right\}^{\mathrm{T}}\{\alpha\}, \quad \tau_{\theta z}^{n}=\frac{G_{n}}{r} \frac{\partial w_{n}}{\partial \theta}=G_{n}\left\{S_{\theta}^{n}\right\}^{\mathrm{T}}\{\alpha\}, \quad 1 \leq n \leq N
$$

where

$$
S_{r i}^{n}=\lambda_{i} r^{\lambda_{i}-1} f_{i}^{n}(\theta), \quad S_{\theta i}^{n}=r^{\lambda_{i}-1} f_{i}^{n^{\prime}}(\theta)
$$

Inserting Eq.(6) into Eq.(2b) gives the total complementary energy in the $C$-region, which can be expressed in terms of the singularity coefficients $\{\alpha\}$ as

Here

$$
\Pi_{C}=\frac{1}{2}\{\alpha\}^{\mathrm{T}}[R]\{\alpha\}
$$

$$
[R]=\sum_{n=1}^{N} G_{n} \iint_{\Omega_{C n}}\left(\left[S_{r}^{n}\right]^{\mathrm{T}}\left[S_{r}^{n}\right]+\left[S_{\theta}^{n}\right]^{\mathrm{T}}\left[S_{\theta}^{n}\right]\right) r \mathrm{~d} r \mathrm{~d} \theta
$$

Since the $C$-region is chosen to be circular, the double integrals can be evaluated separately. Owing to the orthogonal property of the terms in the Williams expansion, the matrix $[R]$, the inverse of which will be used later, turns out to be a diagonal matrix for which all of the diagonal elements can be integrated easily to give the simple closed form solution

$$
R_{i i}=\sum_{n=1}^{N} \frac{G_{n} \lambda_{i}\left|\theta_{n+1}-\theta_{n}\right|}{2} r^{2 \lambda_{i}}\left[\left(a_{i}^{n}\right)^{2}+\left(b_{i}^{n}\right)^{2}\right]
$$

This neat result justifies the earlier choice of the $C$-region as being circular.

The first variation of Eq.(7), which will be used later, is simply

$$
\delta \Pi_{C}=\{\delta \alpha\}^{\mathrm{T}}[R]\{\alpha\}
$$




\subsection{The Plane Stress Case}

It is well known that the complex stress function in each material for plane elasticity can be expressed in the Williams expansion form

$$
\begin{aligned}
& U_{n}=\sum_{i=1}^{\infty} \alpha_{i} r^{\lambda_{i}+1} F_{i}^{n}(\theta) \\
& F_{i}^{n}(\theta)=A_{i}^{n} \sin \left[\left(\lambda_{i}+1\right) \theta\right]+B_{i}^{n} \cos \left[\left(\lambda_{i}+1\right) \theta\right]+C_{i}^{n} \sin \left[\left(\lambda_{i}-1\right) \theta\right]+D_{i}^{n} \cos \left[\left(\lambda_{i}-1\right) \theta\right] \\
& , 1 \leq n \leq N
\end{aligned}
$$

Like the anti-plane case, using the surface conditions and the material interface conditions enables all of the coefficients $A_{i}^{n}, B_{i}^{n}, C_{i}^{n}$ and $D_{i}^{n}$ in the different materials to be expressed in terms of a single coefficient, namely, $\alpha_{i}$. For brevity, the superscript $n$ is omitted from $F_{i}^{n}$ below. The corresponding stress vector in the $C$-region can then be written

$$
\left\{\begin{array}{l}
\sigma_{r i} \\
\sigma_{\theta i} \\
\tau_{r \theta i}
\end{array}\right\}=\alpha_{i} r^{\lambda_{i}-1}\left\{\begin{array}{c}
F_{i}^{\prime \prime}+\left(\lambda_{i}+1\right) F_{i} \\
\lambda_{i}\left(\lambda_{i}+1\right) F_{i} \\
-\lambda_{i} F_{i}^{\prime}
\end{array}\right\}
$$

If the $i$-th term of a stress component is denoted by $\alpha_{i} T_{i}$, then in the case of complex $\lambda_{i}$ there must be another term $\bar{\alpha}_{i} \bar{T}_{i}$ that corresponds to $\bar{\lambda}_{i}$, where the overbar denotes complex conjugate. Combining each pair of such terms yields the two real terms

$$
\alpha_{i} T_{i}+\bar{\alpha}_{i} \bar{T}_{i}=2 T_{R i} \alpha_{R i}-2 T_{I i} \alpha_{I i}
$$

where the subscripts $R$ and $I$ indicate the real and imaginary parts, respectively.

Using this property and rearranging the expansion series enables a $k$ term singular solution for plane problems to be written in the matrix form

$$
\{\sigma\}=[F]\{\alpha\}
$$

Inserting Eq.(14) into Eq.(3b) yields the total complementary energy as Eq.(7) with

$$
[R]=\int_{\Omega_{C}}[F]^{\mathrm{T}}[C][F] r \mathrm{~d} r \mathrm{~d} \theta
$$

Again, the double integrals can be evaluated separately, with the integral with respect to $r$ being obtained in a closed form and the integral with respect to $\theta$ being obtained by a suitable numerical integration. Unlike the anti-plane case, the matrix $[R]$ is no longer a diagonal matrix, but it is still a positive definite one. The first variation of the total complementary energy for the plane stress case is again given by Eq.(10).

\section{THE POTENTIAL ENERGY REGION}

In the potential energy region, the displacement-method-based FEMOL is employed, which will be briefly illustrated for the anti-plane case and directly applied to the plane stress case without detailed derivation. For complete information, see Ref.[3, 4].

\subsection{Element Mapping}

Figure 1(b) shows a typical FEMOL mesh used in this study. The element mapping is common to both the anti-plane and plane stress cases. Let $\tilde{p}$ denote the polynomial degree used for element mapping. Figure 2 shows a typical quadratic element mapping $(\tilde{p}=2)$ in FEMOL from the local $(\xi, \eta)$ space to the global $(x, y)$ space, where $L_{i}(i=1,2, \cdots, \tilde{p}+1)$ indicates the $i$-th nodal line, $S_{j}(j=1,2)$ indicates the $j$-th end-side and accordingly the intersection of $L_{i}$ and $S_{j}$ is denoted by node $i j$. Although in the present study only straight mesh lines are used, in general curved lines are equally applicable when needed. The most-commonly-used element mapping is the parametric mapping

$$
x=\sum_{i=1}^{\tilde{p}+1} N_{i}(\xi) x_{i}(\eta), \quad y=\sum_{i=1}^{\tilde{p}+1} N_{i}(\xi) y_{i}(\eta)
$$




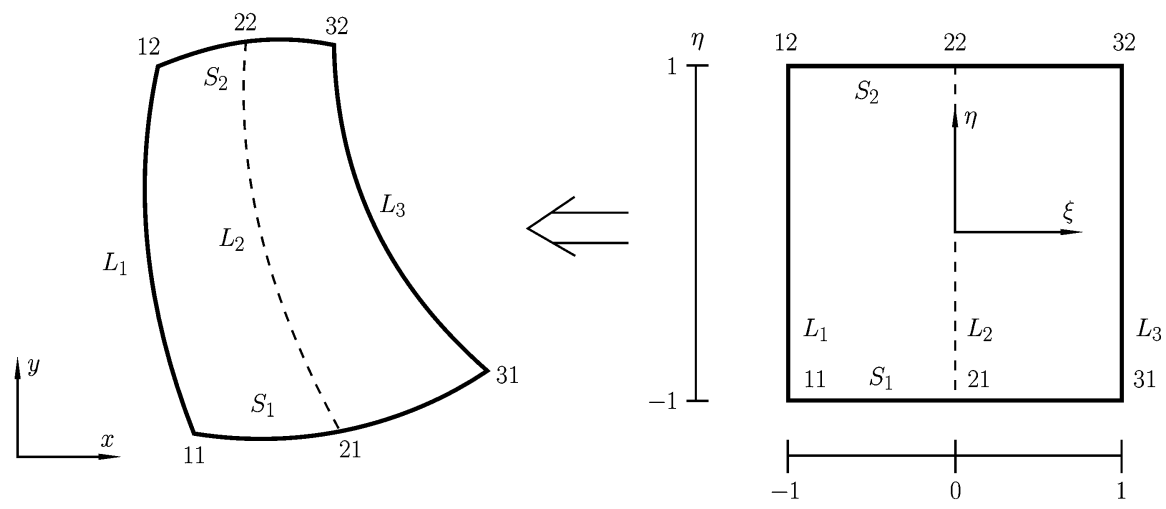

Fig. 2. Quadratic FEMOL element mapping.

Here $N_{i}(\xi)$ is a Lagrange interpolation polynomial of degree $\tilde{p}$ defined in standard interval $[-1,1]$. $\left(x_{i}(\eta), y_{i}(\eta)\right)$ defines parametrically the $i$-th nodal line curve which in the present study is simply a straight line.

The above mapping maps an element end-side to a parametric polynomial curve of degree $\tilde{p}$ while geometrically not conforming with the circular boundary of the $C$-region when the element end-side is connected to the $C$-region. To remove the error introduced by this, blending function interpolation is used to construct a conforming element mapping

$$
\left\{\begin{array}{l}
x \\
y
\end{array}\right\}=\sum_{i=1}^{\tilde{p}+1} \tilde{N}_{i}(\xi)\left\{\begin{array}{l}
\tilde{x}_{i}(\eta) \\
\tilde{y}_{i}(\eta)
\end{array}\right\}+\sum_{j=1}^{\hat{p}+1} \hat{N}_{j}(\eta)\left\{\begin{array}{c}
\hat{x}_{j}(\xi) \\
\hat{y}_{j}(\xi)
\end{array}\right\}-\sum_{i=1}^{\tilde{p}+1} \sum_{j=1}^{\hat{p}+1} \tilde{N}_{i}(\xi) \hat{N}_{j}(\eta)\left\{\begin{array}{l}
x_{i j} \\
y_{i j}
\end{array}\right\}
$$

Here, $\tilde{N}_{i}(\xi)$ and $\hat{N}_{j}(\eta)$ are respectively Lagrange interpolation polynomials in the $\xi$-and $\eta$-directions, $\left(\tilde{x}_{i}(\eta), \tilde{y}_{i}(\eta)\right)$ denotes the $i$-th nodal line curve, $\left(\hat{x}_{j}(\xi), \hat{y}_{j}(\xi)\right)$ denotes the $j$-th end-side curve and $\left(x_{i j}, y_{i j}\right)$ denotes the coordinates of node $i j$. Note that the above mapping is exact even for the linear interpolation $\tilde{p}=\hat{p}=1$, which is exclusively used in this paper. For an end-side curve that is required to be a circular arc, the representation used is

$$
\hat{x}_{i}=x_{c}+R \cos \left(\phi_{1} \xi+\phi_{2}\right), \quad \hat{y}_{i}=y_{c}+R \sin \left(\phi_{1} \xi+\phi_{2}\right)
$$

where $x_{c}$ and $y_{c}$ are the coordinates of the centre of the arc, $R$ is the radius of the arc and $\phi_{1}$ and $\phi_{2}$ are appropriate coefficients locating the arc segment.

For convenience and clarity, the mapping defined by Eq.(16) is called parametric mapping and that defined by Eq.(17) exact mapping. The exact mapping enables a seamless connection between the $P$ and $C$-regions and hence is preferable. With the element mapping defined, the Jacobians of the element area, nodal line segment and end-side segment can be evaluated respectively as

$$
J=x_{\xi} y_{\eta}-x_{\eta} y_{\xi}, \quad J_{L}=\sqrt{\left(x_{\eta}\right)^{2}+\left(y_{\eta}\right)^{2}}, \quad J_{S}=\sqrt{\left(x_{\xi}\right)^{2}+\left(y_{\xi}\right)^{2}}
$$

where ()$_{\xi}=\partial() / \partial \xi,()_{\eta}=\partial() / \partial \eta$.

\subsection{Element Displacement Functions}

The element displacement trial functions can be expressed in a unified matrix form for both anti-plane and plane cases as

$$
\{u\}=[N]\{d\}^{e}
$$

Here $\{u\}$ is the element displacement vector

$$
\{u\}=w \quad \text { for the anti-plane case; } \quad\{u\}=\left\{\begin{array}{ll}
u_{x} & u_{y}
\end{array}\right\}^{\mathrm{T}} \quad \text { for the plane stress case }
$$

$[N]$ is the element shape function matrix

$$
\begin{aligned}
& {[N]=\left[[N]_{1}[N]_{2}, \ldots,[N]_{p+1}\right]} \\
& {[N]_{i}=N_{i}(\xi) \quad \text { for the anti-plane case }} \\
& {[N]_{i}=N_{i}(\xi)[I]_{2 \times 2} \quad \text { for the plane stress case }, \quad i=1,2, \ldots, p+1}
\end{aligned}
$$


and $\{d\}^{e}$ is the element nodal line displacement vector

$$
\begin{aligned}
& \{d\}^{e}=\left\{\begin{array}{ll}
\{d\}_{1}^{\mathrm{T}}\{d\}_{2}^{\mathrm{T}} & \ldots\{d\}_{p+1}^{\mathrm{T}}
\end{array}\right\}^{\mathrm{T}} \\
& \{d\}_{i}^{\mathrm{T}}=w_{i} \quad \text { for the anti-plane case } \quad i=1,2, \ldots, p+1 \\
& \{d\}_{i}^{\mathrm{T}}=\left\{u_{x i}(\eta) u_{y i}(\eta)\right\}^{\mathrm{T}} \quad \text { for the plane stress case }
\end{aligned}
$$

Note that the polynomial degree $p$ used for the element displacement is allowed to be different from the $\tilde{p}$ and $\hat{p}$ used for the element geometric mapping.

\subsection{Strains and Stresses}

For the anti-plane case, the shear strains are the derivatives with respect to $x$ and $y$ which can be expressed as

with

$$
\left\{\begin{array}{l}
\frac{\partial w}{\partial x} \\
\frac{\partial w}{\partial y}
\end{array}\right\}=\frac{1}{J}\left[\begin{array}{cc}
y_{\eta} & -y_{\xi} \\
-x_{\eta} & x_{\xi}
\end{array}\right]\left\{\begin{array}{l}
w_{\xi} \\
w_{\eta}
\end{array}\right\}
$$

$$
w_{\xi}=\frac{\partial w}{\partial \xi}=\left[N^{\prime}\right]\{d\}^{e}, \quad w_{\eta}=\frac{\partial w}{\partial \eta}=[N]\left\{d^{\prime}\right\}^{e}
$$

where the primes represent derivatives with respect to $\xi$ or $\eta$, respectively.

For the plane stress case, by using the strain-displacement relationship and some matrix manipulation, the strain vector can be expressed in the matrix form

$$
\{\varepsilon\}=\left[E_{1}\right]\{d\}^{e}+\left[E_{2}\right]\left\{d^{\prime}\right\}^{e}
$$

Using the stress-strain relation yields the stress vector in the form

$$
\{\sigma\}=\left[S_{1}\right]\{d\}^{e}+\left[S_{2}\right]\left\{d^{\prime}\right\}^{e}
$$

\subsection{Total Potential Energy Functional}

First consider the anti-plane case. By inserting Eqs.(20) and (21) into Eq.(2a), the element total potential energy functional can be obtained as the following form

$$
\Pi_{P}^{e}=\frac{1}{2} \int_{-1}^{1}\left(\left\{d^{\prime}\right\}^{e \mathrm{~T}}[A]^{e}\left\{d^{\prime}\right\}^{e}+2\left\{d^{\prime}\right\}^{e \mathrm{~T}}[B]^{e}\{d\}^{e}+\{d\}^{e \mathrm{~T}}[C]^{e}\{d\}^{e}\right) \mathrm{d} \eta-\sum_{j=1}^{2}\{d\}^{e \mathrm{~T}}\{P\}_{j}^{e}
$$

where

$$
\begin{array}{ll}
{[A]^{e}=\int_{-1}^{1} a(\xi, \eta)[N]^{\mathrm{T}}[N] \mathrm{d} \xi, \quad a(\xi, \eta)=\frac{x_{\xi}^{2}+y_{\xi}^{2}}{J}} \\
{[B]^{e}=\int_{-1}^{1} b(\xi, \eta)[N]^{\mathrm{T}}\left[N^{\prime}\right] \mathrm{d} \xi,} & b(\xi, \eta)=-\frac{x_{\xi} x_{\eta}+y_{\xi} y_{\eta}}{J} \\
{[C]^{e}=\int_{-1}^{1} c(\xi, \eta)\left[N^{\prime}\right]^{\mathrm{T}}\left[N^{\prime}\right] \mathrm{d} \xi,} & c(\xi, \eta)=\frac{x_{\eta}^{2}+y_{\eta}^{2}}{J} \\
\left\{P_{j}\right\}^{e}=\int_{-1}^{1}[N]^{\mathrm{T}} \bar{q}_{S_{j}} J_{S_{j}} \mathrm{~d} \xi, & \left.\bar{q}_{S_{j}} \equiv \bar{q}_{z}\right|_{S_{j}}, \quad \eta=\eta_{j}, \quad j=1,2
\end{array}
$$

The first variation of the element functional (23) with integration by parts yields

$$
\delta \Pi_{P}^{e}=-\int_{-1}^{1}\{\delta d\}^{e \mathrm{~T}}\left([A]^{e}\left\{d^{\prime \prime}\right\}^{e}+[G]^{e}\left\{d^{\prime}\right\}^{e}+[H]^{e}\{d\}^{e}\right) \mathrm{d} \eta+\sum_{j=1}^{2} \eta_{j}\left\{\delta d\left(\eta_{j}\right)\right\}^{e \mathrm{~T}}\left(\left\{Q_{j}\right\}^{e}-\eta_{j}\{P\}_{j}^{e}\right)
$$

where

$$
\begin{array}{ll}
{[G]^{e}=\left[A^{\prime}\right]^{e}+[B]^{e}-[B]^{e \mathrm{~T}},} & {[H]^{e}=\left[B^{\prime}\right]^{e}-[C]^{e}} \\
\left\{Q_{j}\right\}^{e}=[A]^{e}\left\{d^{\prime}\right\}+[B]^{e}\{d\}^{e}, & \eta=\eta_{j}, \quad j=1,2 \\
{\left[A^{\prime}\right]^{e}=\int_{-1}^{1} \frac{\partial a}{\partial \eta}[N]^{\mathrm{T}}[N] \mathrm{d} \xi,} & {\left[B^{\prime}\right]^{e}=\int_{-1}^{1} \frac{\partial b}{\partial \eta}[N]^{\mathrm{T}}\left[N^{\prime}\right] \mathrm{d} \xi}
\end{array}
$$


For the plane stress case, although the coefficient matrices have different components, the element functional and its first variation can be expressed in exactly the form of Eqs.(23) and (24), respectively.

Standard element assembly procedures lead to the global functional, which takes exactly the same form as the element one Eq.(23) except that the superscript $e$ is removed. Similarly, the first variation of the global functional also takes the same form as that in Eq.(24) except that the superscript $e$ is removed.

\section{RESULTING EQUATION SYSTEM}

The interface term $H_{P C}$ can be written in terms of $\{d\}$ and $\{\alpha\}$ as

$$
H_{P C}=\{\alpha\}^{\mathrm{T}}[W]\{d\}
$$

where

$$
\begin{array}{ll}
{[W]=\sum_{n=1}^{N} G_{n} \int_{\Gamma_{P C n}}\left\{S_{r}^{n}\right\}[N] r \mathrm{~d} \theta \quad \text { for the anti-plane case }} \\
{[W]=\sum_{n=1}^{N} \int_{\Gamma_{P C n}}\{F\}^{\mathrm{T}}[T][N] r \mathrm{~d} \theta \quad \text { for the plane stress case }}
\end{array}
$$

where $[T]$ is an appropriate coordinate transformation matrix. The first variation of Eq.(25) is

$$
\delta H_{P C}=\{\delta \alpha\}^{\mathrm{T}}[W]\{d\}+\{\delta d\}^{\mathrm{T}}[W]^{\mathrm{T}}\{\alpha\}
$$

Equations (10), (24) and (26) sum to the first variation of the total mixed energy functional $\delta \Pi$ (see Eq.(1)) and the corresponding stationary condition yields a mixed equation system that consists of ODEs, the associated boundary conditions (BCs) and a set of algebraic equations (AEs) as follows

$$
\begin{array}{ll}
\text { ODEs: } & -1<\eta<1, \quad[A]\left\{d^{\prime \prime}\right\}+[G]\left\{d^{\prime}\right\}+[H]\{d\}+\{F\}=\{0\} \\
\text { BCs: } & \eta=\eta_{j}, \quad j=1,2, \quad d_{m}=\bar{d}_{m} \quad \text { or } \quad Q_{j m}=\eta_{j} P_{j m}, \quad \eta_{j} \notin \Gamma_{P C} \\
\text { BCs: } \quad \eta=\eta_{j}, \quad j=1,2, \quad Q_{j m}=\eta_{j}[W]^{\mathrm{T}}\{\alpha\}, \quad \eta_{j} \in \Gamma_{P C} \\
\text { AEs : } & {[R]\{\alpha\}=[W]\{d\}, \quad \eta_{j} \in \Gamma_{P C}}
\end{array}
$$

Note that Eq.(27b) expresses the standard FEMOL BCs and that Eq.(27c) is coupled with the system of AEs in Eq.(27d). Also note that the stress parameters in $\{\alpha\}$ are closely related to the SIFs and will be given directly as part of the solution. This mixed equation system is not in the standard ODE form which standard ODE solvers such as COLSYS can accept. The singularity removal technique (SRT) is now proposed to overcome this difficulty.

From Eq.(27d) the stress parameter vector can be solved as

$$
\{\alpha\}=[R]^{-1}[W]^{\mathrm{T}}\{d\}
$$

This step presents no difficulty and is computationally easy since $[R]$ is a simple diagonal matrix in the anti-plane case and a small-sized positive-definite matrix in the plane stress case. Eliminating $\{\alpha\}$ from Eq.(27c) by using Eq.(28) changes Eq.(27c) into the standard BC expression

$$
\begin{aligned}
& Q_{j m}=\eta_{j}\left\{P_{j}\right\} \\
& \left\{P_{j}\right\}=[W]^{T}[R]^{-1}[W]\left\{d\left(\eta_{j}\right)\right\}, \quad \eta_{j} \in \Gamma_{P C}
\end{aligned}
$$

As a result, the final equation system requiring solution is the standardized ODE system formed by Eqs.(27a), (27b) and (29a), which can be solved directly and efficiently by COLSYS.

Compared with the standard FEMOL formulation, the only difference is in the load vector $\left\{P_{j}\right\}$ on the right-hand side of Eq.(29a). This is derived from the given boundary traction forces in the standard formulation but is now given by Eq.(29b) instead, which may well be viewed as a variationally equivalent traction vector implicitly derived from the action of the singular stresses in $\Omega_{C}$ on the regular region $\Omega_{P}$. In addition to the standardization of the ODE system, a more significant effect of eliminating the AEs in Eq.(27d) is that the standardized ODE system renders a smooth solution $\{d\}$ defined on the 
regular region $\Omega_{P}$ with the singular region $\Omega_{C}$ effectively removed, see Fig.1(c). In other words, the problem which was once singular has now become a standard and regular FEMOL problem with the singular region, along with the singular stresses residing in it, removed from the solution domain. To obtain the desired SIFs after solving the standardized ODE system, it is simply necessary to insert $\{d\}$ back into Eq.(28).

Compared with the sub-region FEM, the replacement of FEM by FEMOL overcomes, to a great extent, the crucial FEM difficulty of how large the singular region $\Omega_{C}$ should be. For instance, when $\Omega_{C}$ is small a few singular terms are sufficient for stresses, but the solution on $\Omega_{P}$ tends to become singular. Hence sophisticated mesh refinement is needed around $\Omega_{C}$ when using FEM, especially in the radial direction. This problem is not expected to be so crucial when using FEMOL plus the singularity removal technique when a favorable choice of radial line meshes is made. The self-adaptivity capability built into the solver COLSYS will capture any possible badly behaved solution in the radial line direction. On the other hand, in the circular direction the solution is relatively smooth and hence demands less adaptivity, so that it can be effectively approximated by the polynomial shape functions assumed in FEMOL elements. By adapting itself to produce an ODE solution satisfying the user-specified error tolerance, FEMOL provides not only improved accuracy but also greater credibility for the computed results and greater ease for both the analyst and the computer itself.

\section{NUMERICAL EXAMPLES}

A number of numerical examples are now given to evaluate the performance of the proposed method using the following notation: $r_{0}$ - radius of the singular region; $k$ - number of leading terms used in the singular solution; $p$ — polynomial degree used for element displacements; $\tilde{p}$ — polynomial degree used for element geometry mapping; Tol — tolerance specified for ODE solutions; $E, G, \nu$ — Young's modulus, shear modulus and the Poisson's ratio; $K_{\mathrm{I}}, K_{\mathrm{II}}, K_{\mathrm{III}}$ - stress intensity factors.

For the anti-plane case the tolerances for the solver COLSYS are imposed on both displacements and their derivatives, whereas for the plane stress case the tolerances are only imposed on displacements. All examples were computed on a Pentium $100 \mathrm{MHz}$ computer.

Example 1. L-shaped Laplace problem

As the first example, the Laplace equation defined on the L-shaped domain shown in Fig.3 is considered. The requirement was to compute the important factors $\alpha_{1}$ and $\alpha_{2}$ in Eq.(5). Two leading terms $(k=2)$ were taken in the singular region (the term corresponding to $\alpha_{0}$ does not produce any complementary energy) and six FEMOL elements were used.

Table 1 lists the computed results for: different radii of the singular region; different polynomial degrees of element displacements; and both standard mapping and exact mapping on $\Gamma_{P C}$. It can be seen that for linear elements the exact mapping improved the results substantially, whereas for higher-order elements the two mapping techniques gave similar accuracy. It can also be seen that the results depend very little on the radius of the singular region, even in the very small and very large cases, i.e. $r_{0}=0.1$

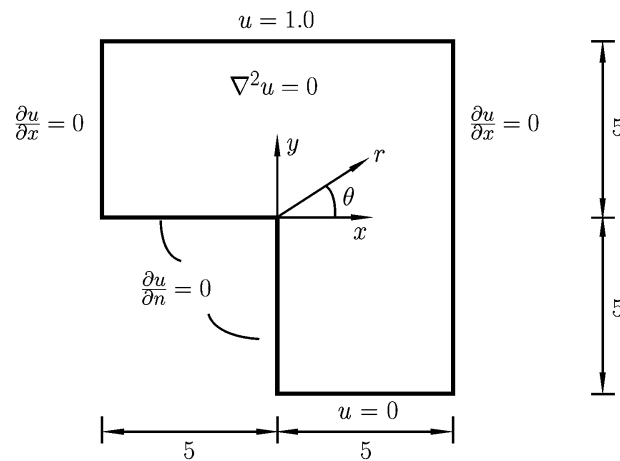

(a) problem definition

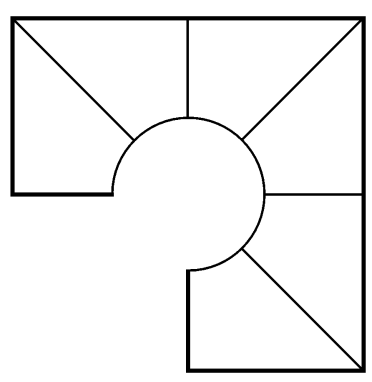

(b) FEMOL mesh

Fig. 3. L-shaped Laplace problem. 
Table 1. Computed results $(k=2$, Tol $=0.01 \%)$

\begin{tabular}{cccccccc}
\hline \multirow{2}{*}{$\Gamma_{P C}$} & $r_{0}$ & \multicolumn{2}{c}{$p=1$} & \multicolumn{2}{c}{$p=2$} & \multicolumn{2}{c}{$p=3$} \\
& & $\alpha_{1}$ & $\alpha_{2}$ & $\alpha_{1}$ & $\alpha_{2}$ & $\alpha_{1}$ & $\alpha_{2}$ \\
\hline \multirow{5}{*}{$p=1$} & 0.151558 & 0.022525 & 0.154867 & 0.025059 & 0.154564 & 0.025142 \\
& 1.0 & 0.151385 & 0.022680 & 0.154658 & 0.025079 & 0.154596 & 0.025135 \\
& 2.0 & 0.150956 & 0.022847 & 0.154626 & 0.025088 & 0.154597 & 0.025135 \\
& 3.0 & 0.150495 & 0.023007 & 0.154611 & 0.025091 & 0.154597 & 0.025135 \\
& 4.0 & 0.149874 & 0.023209 & 0.154604 & 0.025092 & 0.154587 & 0.025136 \\
& 4.5 & 0.149402 & 0.023358 & 0.154556 & 0.025103 & 0.154540 & 0.025146 \\
exact & 0.1 & 0.153726 & 0.023118 & 0.154762 & 0.025029 & 0.154556 & 0.025140 \\
& 1.0 & 0.154604 & 0.023630 & 0.154672 & 0.025072 & 0.154589 & 0.025133 \\
& 2.0 & 0.154942 & 0.024055 & 0.154654 & 0.025087 & 0.154591 & 0.025133 \\
& 3.0 & 0.154967 & 0.024406 & 0.154653 & 0.025098 & 0.154591 & 0.025133 \\
& 4.0 & 0.154549 & 0.024748 & 0.154659 & 0.025106 & 0.154581 & 0.025134 \\
& 4.5 & 0.154078 & 0.024965 & 0.154617 & 0.025122 & 0.154534 & 0.025143 \\
& best known $^{[4]}$ & 0.1546 & 0.02513 & 0.1546 & 0.02513 & 0.1546 & 0.02513 \\
\hline
\end{tabular}

and 4.5, respectively. Including more terms was found to be unnecessary, whereas using higher- order elements was beneficial.

Example 2. Bimaterial anti-plane disk with a notch ${ }^{[20]}$

This example is anti-plane deformation of a disk with a notch, see Fig.4. For convenience, the nondimensional radius of the disk is taken as $R=10$ and other related data are $\alpha=90^{\circ}, G_{1}: G_{2}=1: 10$, and $\theta_{1}=\theta_{2}$. The displacement in the disk used only three terms of Eq.(5). The coefficients $a_{i}^{1}$ and $b_{i}^{1}$ in the first material were determined by:

For $\left|\cos \left(\lambda_{i} \theta_{1}\right)\right| \leq\left|\sin \left(\lambda_{i} \theta_{1}\right)\right|$, then $b_{i}^{1}=1, a_{i}^{1}=\cos \left(\lambda_{i} \theta_{1}\right) / \sin \left(\lambda_{i} \theta_{1}\right)$, whereas otherwise $a_{i}^{1}=1$, $b_{i}^{1}=\sin \left(\lambda_{i} \theta_{1}\right) / \cos \left(\lambda_{i} \theta_{1}\right)$ and the coefficients $a_{i}^{2}$ and $b_{i}^{2}$ were calculated from the interface conditions. To complete the assigned data, the following two alternative sets of three factors were assumed

case $1: \alpha_{1}=1.0, \quad \alpha_{2}=0.1, \quad \alpha_{3}=0.01, \quad$ case $2: \alpha_{1}=0.01, \quad \alpha_{2}=0.1, \quad \alpha_{3}=1.0$

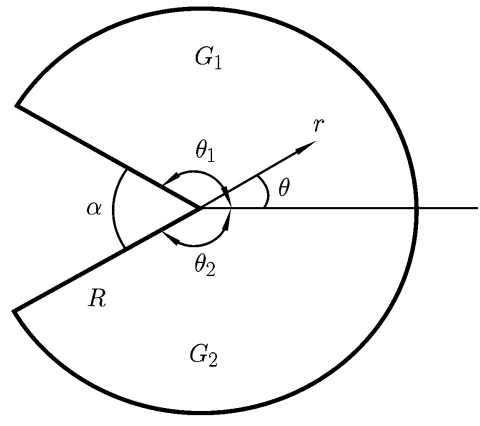

(a) problem definition

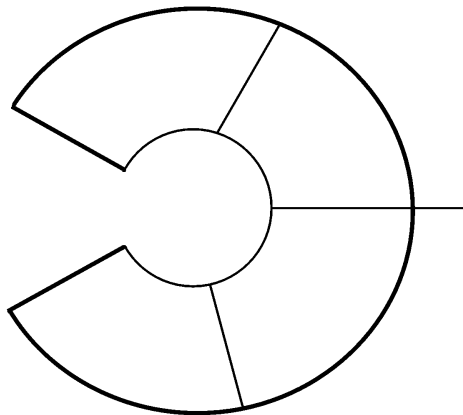

(b) FEMOL mesh

Fig. 4. Bimaterial anti-plane disk with notch.

Table 2. Computed results for case 1

\begin{tabular}{cccc}
\hline$r_{0}$ & $\alpha_{1}$ & $\alpha_{2}$ & $\alpha_{3}$ \\
\hline 0.5 & 0.9999986 & 0.1000037 & 0.0100029 \\
1.0 & 1.0000005 & 0.1000035 & 0.0100031 \\
2.0 & 1.0000005 & 0.1000035 & 0.0100033 \\
4.0 & 1.0000003 & 0.1000036 & 0.0100035 \\
6.0 & 1.0000006 & 0.1000036 & 0.0100036 \\
8.0 & 1.0000006 & 0.1000036 & 0.0100037 \\
9.0 & 1.0000006 & 0.1000036 & 0.0100037 \\
exact & 1.0 & 0.1 & 0.01 \\
\hline
\end{tabular}


Table 3. Computed results for case 2

\begin{tabular}{cccc}
\hline$r_{0}$ & $\alpha_{1}$ & $\alpha_{2}$ & $\alpha_{3}$ \\
\hline 0.5 & 0.00999945 & 0.1000095 & 1.0002909 \\
1.0 & 0.00999999 & 0.1000037 & 1.0003112 \\
2.0 & 0.00999999 & 0.1000036 & 1.0003315 \\
4.0 & 0.01000000 & 0.1000036 & 1.0003517 \\
6.0 & 0.01000001 & 0.1000036 & 1.0003629 \\
8.0 & 0.01000001 & 0.1000036 & 1.0003693 \\
9.0 & 0.01000001 & 0.1000036 & 1.0003710 \\
exact & 0.01 & 0.1 & 1.0 \\
\hline
\end{tabular}

The displacement on the outer boundary was determined accordingly and was used as the boundary condition for this example. In computation, four FEMOL elements with $p=4$ and exact mapping were used, see Fig.4(b) and the tolerance for COLSYS was set as Tol=0.001\%. The computed results are listed in Tables 2 and 3 respectively, from which satisfactory accuracy is evident.

Example 3. Plane disk with a mixed-mode crack

This example is similar to example 2 except that the present example is for a single material crack and in plane stress. For convenience, the non-dimensional data were taken as $E=1.0, \nu=0.25$, $R=10.0$ and $\alpha=0$. In the disk, three terms of the Williams expansion (11) were taken, from which the displacements on the outer boundary were determined and used as the boundary condition for this example. The first three eigenvalues are $0.5,0.5$ and 1.0 respectively, and the corresponding eigenfunctions are given by replacing the coefficients $\left\{A_{i}, B_{i}, C_{i}, D_{i}\right\}$ in Eq.(11) with $\{0,1 / 3,0,1\}$, $\{-1,0,1,0\}$ and $\{0,-1,0,1\}$, respectively. It is worth mentioning that the fourth eigenvalue is also 1.0 , with eigenfunction coefficients $\{0,0,1,0\}$, but was not selected because it does not contribute any stress. To complete the given data, two sets of factors were assumed, namely,

case $1: \alpha_{1}=20.0, \quad \alpha_{2}=10.0, \quad \alpha_{3}=20.0, \quad$ case $2: \alpha_{1}=10.0, \quad \alpha_{2}=20.0, \quad \alpha_{3}=40.0$

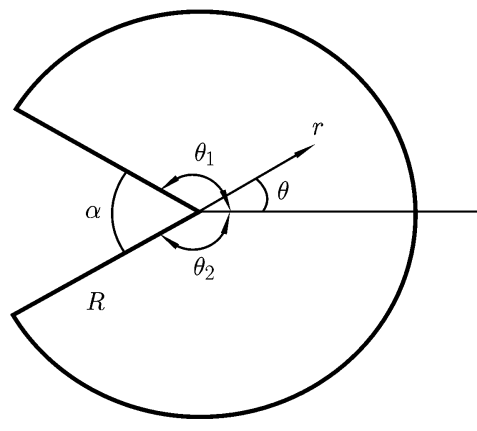

(a) problem definition

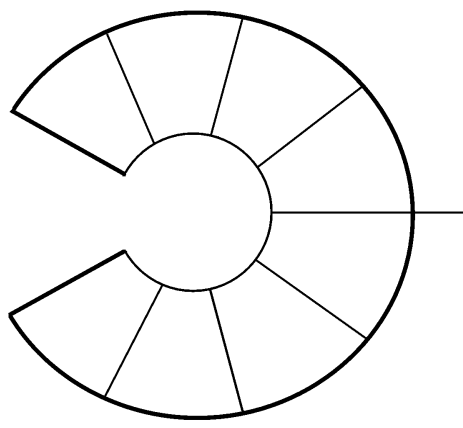

(b) FEMOL mesh

Fig. 5. Plane disk with crack.

Table 4. Computed results for case 1

\begin{tabular}{cccc}
\hline$r_{0}$ & $\alpha_{1}$ & $\alpha_{2}$ & $\alpha_{3}$ \\
\hline 0.001 & 19.987415 & 9.998942 & 20.074443 \\
0.01 & 19.979297 & 10.000717 & 20.062736 \\
1.0 & 19.999574 & 10.000018 & 19.999892 \\
3.0 & 19.999167 & 9.9999990 & 19.999928 \\
5.0 & 19.999058 & 10.000021 & 19.999954 \\
7.0 & 19.998657 & 10.000026 & 19.999964 \\
9.0 & 19.998177 & 10.000030 & 19.999970 \\
exact & 20 & 10 & 20 \\
\hline
\end{tabular}


Table 5. Computed results for case 2

\begin{tabular}{cccc}
\hline$r_{0}$ & $\alpha_{1}$ & $\alpha_{2}$ & $\alpha_{3}$ \\
\hline 0.001 & 9.999399 & 19.999941 & 39.998788 \\
0.01 & 9.999432 & 19.999917 & 39.999581 \\
1.0 & 9.999293 & 20.000035 & 39.999860 \\
3.0 & 9.998547 & 19.999998 & 39.999893 \\
5.0 & 9.997920 & 20.000043 & 39.999925 \\
7.0 & 9.997076 & 20.000052 & 39.999946 \\
9.0 & 9.996082 & 20.000060 & 39.999963 \\
exact & 10 & 20 & 40 \\
\hline
\end{tabular}

In computation, eight FEMOL elements with $p=4$ and $\tilde{p}=2$ were used, see Fig.5(b), and the tolerance for COLSYS was set as Tol $=0.1 \%$. The computed results are listed in Tables 4 and 5 and once again the accuracy is satisfactory. It is worth noting that even for an extremely small singular region, e.g. $r_{0}=0.001$, the results are still quite acceptable.

Example 4. Mixed-mode crack in plane stress

The problem and FEMOL mesh used are shown in Fig.6, and the non-dimensional data were $E=1.0$, $\nu=0.25, w=7$ and $\tau_{0}=1.0$. Like the previous example, the first six eigenvalues $\lambda_{1}, \lambda_{2}, \ldots$ are 0.5 , $0.5,1.0,1.0,1.5$ and 1.5. Arguments identical to those given in the previous example allowed omission of the fourth term (i.e. one of the two with eigenvalue 1.0), so that $k=2,3,5$ implies respectively: the two terms for $\lambda_{1}=\lambda_{2}=0.5$; the three terms for $\lambda_{1}=\lambda_{2}=0.5$ and $\lambda_{3}=1.0$; and the five terms for $\lambda_{1}=\lambda_{2}=0.5, \lambda_{3}=1.0$ and $\lambda_{4}=\lambda_{5}=0.5$. Furthermore, the SIFs are defined as follows:

$$
K_{\mathrm{I}}=\left.\sqrt{2 \pi} \lim _{r \rightarrow 0} r^{\frac{1}{2}} \sigma_{\theta}\right|_{\theta=0}, \quad K_{\mathrm{II}}=\left.\sqrt{2 \pi} \lim _{r \rightarrow 0} r^{\frac{1}{2}} \sigma_{r \theta}\right|_{\theta=0}
$$

In computation, eight FEMOL elements with $p=4$ and $\tilde{p}=2$ were used, see Fig.6(b), and the tolerance for COLSYS was taken as Tol $=0.01 \%$. The computed results are listed in Table 6 , from which it can be seen that the five-term solution produces very considerably better results for relatively large singular regions.

Example 5. V-notch specimen

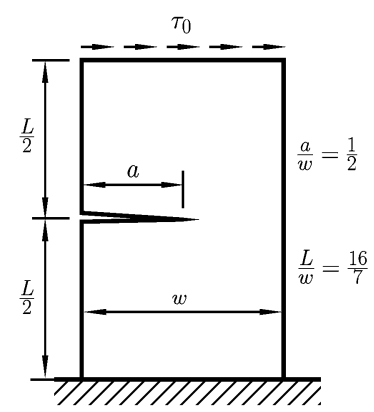

(a) problem definition

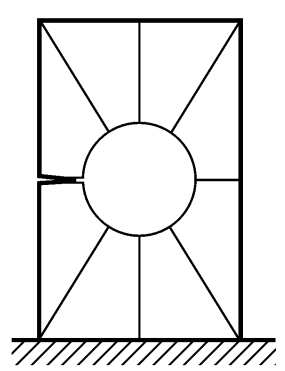

(b) FEMOL mesh

Fig. 6. Mixed mode crack.

Table 6. Computed results for $K_{\mathrm{I}}$ and $K_{\mathrm{II}}$

\begin{tabular}{cccc}
\hline$r_{0}$ & $k=2$ & $k=3$ & $k=5$ \\
\hline 0.001 & $34.001,4.542$ & $33.940,4.541$ & $33.926,4.541$ \\
0.01 & $34.033,4.537$ & $33.927,4.537$ & $33.940,4.539$ \\
0.1 & $33.629,4.509$ & $33.393,4.509$ & $33.885,4.536$ \\
0.5 & $31.160,4.403$ & $31.030,4.402$ & $33.274,4.534$ \\
1.0 & $27.997,4.293$ & $28.116,4.293$ & $32.103,4.521$ \\
2.0 & $22.354,4.172$ & $22.647,4.169$ & $28.568,4.384$ \\
Ref.[21] & $33.20 \leq K_{\text {I }} \leq 34.25, \quad 4.50 \leq K_{\text {II }} \leq 4.79$ \\
\hline
\end{tabular}




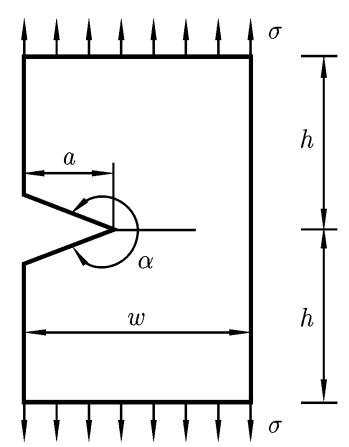

(a) problem definition

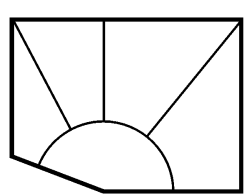

(b) FEMOL mesh

Fig. 7. V-notch specimen in tension.

Table 7. Computed results for $K_{\mathrm{I}}$

\begin{tabular}{ccccc}
\hline$r_{0} / w$ & $p$ & $k=1$ & $k=2$ & $k=3$ \\
\hline \multirow{3}{*}{0.001} & 3 & 3.7346 & 3.7362 & 3.7354 \\
& 4 & 3.7580 & 3.7472 & 3.7611 \\
& 5 & 3.7539 & 3.7492 & 3.7598 \\
0.01 & 3 & 3.7341 & 3.7339 & 3.7403 \\
& 4 & 3.7440 & 3.7482 & 3.7572 \\
& 5 & 3.7484 & 3.7515 & 3.7601 \\
0.1 & 3 & 3.5723 & 3.5722 & 3.7467 \\
& 4 & 3.5789 & 3.5788 & 3.7561 \\
\multicolumn{4}{c}{ Ref.[22]: $K_{\mathrm{I}}=3.766$} & Ref.[23]: $K_{\mathrm{I}}=3.754$
\end{tabular}

Figure 7(a) defines the problem and the non-dimensional data are $h / w=1.0, a / w=0.4, w=1.0$, $\alpha=240^{\circ}, E=1.0, \nu=0.3$ and $\sigma=1.0$. The FEMOL mesh used is shown in Fig.7(b). Exact mapping was used and the tolerance for COLSYS was taken as Tol $=0.01 \%$. Table 7 shows the results computed for different $k^{\prime}, p^{\prime}$ and $r_{0}$ 's. These results agree very well with the solutions from the literature shown ${ }^{[21,22]}$.

\section{CONCLUDING REMARKS}

The sub-region mixed finite element method of lines (FEMOL) has been successfully applied to stress intensity factor (SIF) computations for two-dimensional notch/crack problems. Numerical examples presented in the preceding section have shown that the proposed method is capable of producing highly accurate and reliable solutions with relatively few singular terms and FEMOL elements used. In this new implementation, replacing fully discrete FEM with semi-analytical FEMOL in the original sub-region mixed FEM is a major advance and has led to the following remarkable advantages.

1. The new implementation is still a general method for SIF computation, i.e. it is applicable to twodimensional crack/notch problems with arbitrary domains, arbitrary opening angles, arbitrary multiple materials.

2. The proposed method does not rely on any special means, e.g. a special ordinary differential equation (ODE) solver, because the ODE system derived from FEMOL with the singularity removal technique is a standard linear ODE problem and hence any standard ODE solver can equally be used to obtain accurate and reliable solutions.

3. The semi-analytical feature inherent in FEMOL leads to more accurate solutions in the potential energy region which implies that, on the one hand, the number of FEMOL elements may be decreased for moderate accuracy and, on the other, the solution credibility is increased.

4. Because the solutions in the potential region are no longer singular, the $p$-strategy (i.e. increasing the polynomial degree $p$ in each element with the element mesh fixed) is usually more beneficial and efficient. 
5. Using the favorable radial line mesh makes the solution automatically self-adaptive in radial directions and hence makes the solution much less sensitive to the size of the singular region. Numerical examples have confirmed that roughly the same accuracy is given throughout the range from extremely small to medium-sized singular regions.

6. Another small but important choice is to use circular singular regions which, on the one hand, simplifies the formulation in the singular region and, on the other, makes full use of the orthogonal relationship between eigenfunctions for anti-plane problems. As a result, for anti-plane problems the solutions are nearly independent of the radius of the singular region and of the number of leading terms used in the singular solutions.

\section{References}

[1] Yuan,S., A new semi-discrete method-the finite element method of lines//Proceedings of 1st National Conference on Analytical and Numerical Combined Methods, Hunan, 1990, 132-136 (in Chinese).

[2] Yuan,S. and Gao,J.L., A new computational tool in structural analysis: the finite element method of lines (FEMOL)//Proceedings of International Conference on EPMESC, Macau, 1990, 3(1-3): 517-526.

[3] Yuan,S., The finite element method of lines. Chinese Journal of Numerical Mathematics and Applications, 1993, 15(1): 45-59.

[4] Yuan,S., The Finite Element Method of Lines: Theory and Applications. Beijing-New York: Science Press, 1993.

[5] Ascher,U., Christiansen,J. and Russell,R.D., Collocation software for boundary-value ODEs. ACM Transaction of Mathematical Software, 1981, 7(2): 209-222.

[6] Ascher,U., Christiansen,J. and Russell,R.D., Algorithm 569, COLSYS: collocation software for boundaryvalue ODEs [D2]. ACM Transaction of Mathematical Software, 1981, 7(2): 223-229.

[7] Yuan,S., A general-purpose FEMOL program-FEMOL92. Computational Structural Mechanics and Applications, 1993, 10(1): 118-122 (in Chinese).

[8] Yuan,S., The 'triangular' elements in the finite element method of lines. Science in China, Series A, 1993, 23(5): 552-560 (in Chinese).

[9] Xu,Y.J. and Yuan,S., Complete eigen-solutions for plane notches with multi-materials by the imbedding method. International Journal of Fracture, 1996, 81(4): 373-381.

[10] Xu,Y.J. and Yuan,S., Complete eigensolutions for anti-plane notches with multi-materials by super-inverse iteration. Acta Mechanica Solida Sinica, 1997, 10(2): 157-166.

[11] Xu,Y.J., Yuan,S. and Liu,C.T., The progress on complete engen-solution of two dimensional notch problems. Advances in Mechanics, 2000, 30(2): 216-226 (in Chinese).

[12] Xu,Y.J., Yuan,S. and Liu,C.T., Possible multiple roots for fracture problems. Acta Mechanica Sinica, 1999, 31(5): 618-624 (in Chinese).

[13] Xu,Y.J., Eigenproblem in Fracture Mechanics for Reissner plate. Acta Mechanica Solida Sinica, 2004, 25(2): 225-228 (in Chinese).

[14] Xu,Y.J. and Liu,C.T., The eigenvalues and eigenfunctions in shallow shell fracture analysis. Acta Mechanica Solida Sinica, 2000, 21(3): 256-260 (in Chinese).

[15] Long,Y.Q., Sub-region generalized variational principles in elasticity. Shanghai Journal of Mechanics, 1981, 2(2): 1-9 (in Chinese).

[16] Long,Y.Q., Zhi,B.C. and Yuan,S., Sub-region, sub-item and sub-layer generalized variational principles in elasticity. Proceedings of International Conference on Finite Element Methods, (ed. He Guangqian and Y. K. Cheung), Shanghai, China 1982, 607-609.

[17] Long,Y.Q. Zhi,B.C., Kuang,W.Q. and Shan,J., Sub-region mixed finite element analysis of stress intensity factors. Sinica Mechanica, 1982, 4: 341-353.

[18] Long,Y.Q. and Zhao,Y.Q., Calculation of stress intensity factors in plane problems by the sub-region mixed finite element method. Advances in Engineering Software, 1985, 7(1): 32-35.

[19] Fan,Z. and Long,Y.Q., Sub-region mixed finite element analysis of V-notched plates. International Journal of Fracture, 1992, 56: 333-344.

[20] Xu,Y.J. and Yuan,S., Stress intensity factors calculation in anti-plane fracture problem by orthogonal integral extraction method based on FEMOL. Acta Mechanica Solida Sinica, 2007, 20(1): 87-94.

[21] Stern,M., Becker,E.B. and Dunham,R.S., A contour integral computation of mixed-mode stress intensity factors. International Journal of Fracture, 1976, 12: 359-368.

[22] Gross,B. and Mendelson,A., Plane elastic analysis of V-notched plates. International Journal of Fracture Mechanics, 1972, 8: 267-276.

[23] Long,Y.Q., Introduction of New Finite Element Methods. Beijing: Tsinghua University Press, 1992. 